\title{
Intuitive Exploration of Volumetric Data Using Dynamic Galleries
}

\author{
Daniel Jönsson, Martin Falk and Anders Ynnerman
}

\section{Linköping University Post Print}

\section{Tweet}

N.B.: When citing this work, cite the original article.

Daniel Jönsson, Martin Falk and Anders Ynnerman, Intuitive Exploration of Volumetric Data Using Dynamic Galleries, 2016, IEEE Transactions on Visualization and Computer Graphics, (22), 1, 896-905.

\section{http://dx.doi.org/10.1109/TVCG.2015.2467294}

(C2016 IEEE. Personal use of this material is permitted. However, permission to reprint/republish this material for advertising or promotional purposes or for creating new collective works for resale or redistribution to servers or lists, or to reuse any copyrighted component of this work in other works must be obtained from the IEEE.

\section{http://ieeexplore.ieee.org/}

Postprint available at: Linköping University Electronic Press

http://urn.kb.se/resolve?urn=urn:nbn:se:liu:diva-123054 


\title{
Intuitive Exploration of Volumetric Data Using Dynamic Galleries
}

\author{
Daniel Jönsson, Student Member, IEEE, Martin Falk, Member, IEEE, \\ and Anders Ynnerman, Associate Member, IEEE
}
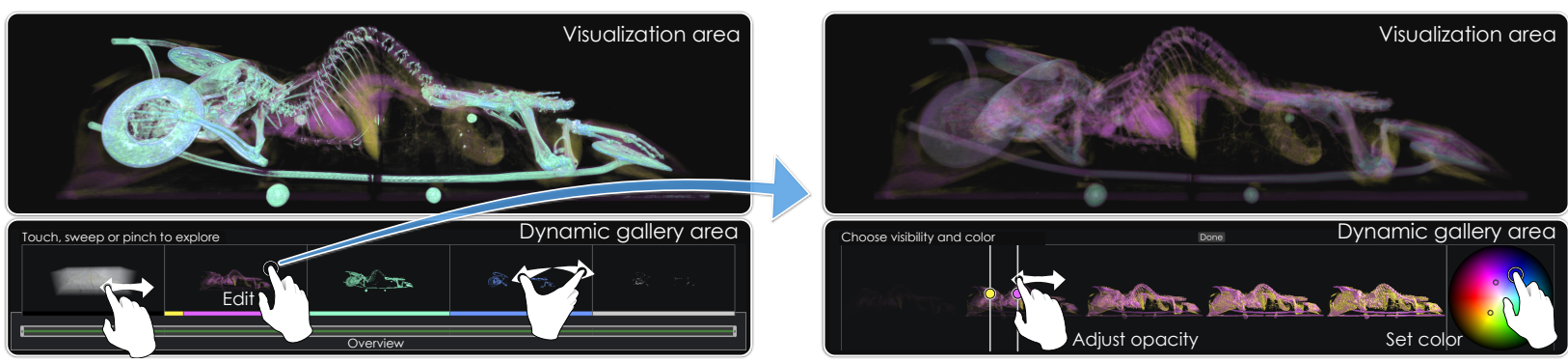

Fig. 1: Left: The top shows the final rendering while the bottom part provides a dynamically generated image gallery of the data domain. Overview and details of the data domain are presented on demand by zooming and panning in the gallery using pinch and swipe touch gestures. Pressing a gallery image enables the user to edit opacity and color of the respective data range. Right: In edit mode, the feature is highlighted in the top by decreasing the opacity of everything else which was found to be essential for novice users. The bottom area enables intuitive editing of opacity and color using a nonlinear mapping function and a simplified color picker.

\begin{abstract}
In this work we present a volume exploration method designed to be used by novice users and visitors to science centers and museums. The volumetric digitalization of artifacts in museums is of rapidly increasing interest as enhanced user experience through interactive data visualization can be achieved. This is, however, a challenging task since the vast majority of visitors are not familiar with the concepts commonly used in data exploration, such as mapping of visual properties from values in the data domain using transfer functions. Interacting in the data domain is an effective way to filter away undesired information but it is difficult to predict where the values lie in the spatial domain. In this work we make extensive use of dynamic previews instantly generated as the user explores the data domain. The previews allow the user to predict what effect changes in the data domain will have on the rendered image without being aware that visual parameters are set in the data domain. Each preview represents a subrange of the data domain where overview and details are given on demand through zooming and panning. The method has been designed with touch interfaces as the target platform for interaction. We provide a qualitative evaluation performed with visitors to a science center to show the utility of the approach.
\end{abstract}

Index Terms-Transfer function, scalar fields, volume rendering, touch interaction, visualization, user interfaces.

\section{INTRODUCTION}

Volume data visualization is an essential tool for exploring 3D scalar fields and has been extensively used in several domains ranging from medical, where a radiologist explores the interior of the human body in order to determine if the patient is healthy, to the public, where museums and science centers are able to show the interior of physical items on display. While the tools used by experts have been extensively developed [24, 15], little effort has gone into making tools for novice users. In the public domain, it has been seen as too daunting for users to handle the exploration tools available today. Instead a visualization expert determines a few presets that the novice user is allowed to select from and the interaction is limited to spatially exploring the data by moving the camera. Sadly, large parts of the volume visualization field therefore becomes inaccessible to the general public.

In order for volume data visualization to reach its full potential and become used by people who are not expert users it is clear that there is a need for an exploration tool that is intuitive enough for a ten year old visiting a museum to use, with little or no instructions. Finding a solution that enables a new user to seamlessly explore volume data is a

- Daniel Jönsson, Martin Falk, and Anders Ynnerman are with Linköping University, Sweden. E-mail:

\{daniel.jonsson, martin.falk, anders.ynnerman\}@liu.se.

Manuscript received 31 Mar. 2015; accepted 1 Aug. 2015; date of publication xx Aug. 2015; date of current version 25 Oct. 2015. For information on obtaining reprints of this article, please send e-mail to:tvcg@computer.org. difficult challenge. One of the most widely used tools today is a transfer function that allows the user to map opacity and color based solely on the intensity of the data. The intensity-based transfer function is conceptually simple and can therefore easily be understood. However, it is extremely hard to know what effect changing a visualization parameter for a certain intensity will have on the resulting visualization. The lack of spatial connection also make it difficult to retrieve the desired visualization since the same intensity value may be located in spatially different locations and there is no way of changing just one of them. Design galleries are at the other end of the spectrum, where previews are computed based on an analysis of the data and shown to the user. The user can select which preview to display in the final rendering. Design galleries have the benefit that the changes made by the user are intuitive and logical, since the spatial context is given by the preview, but it is unclear how to explore parameters that were not initially extracted or how to navigate the multidimensional space. Furthermore, designing the algorithm that extracts parameters for the previews is challenging and domain specific

In this work we introduce a novel method that dynamically generates galleries based on intuitive interaction, and allows the user to drill down to detail without loss of control. The method is intuitive, such that a person with little or no experience can start exploring the data. The method is conceptually simple, such that it is easy to grasp. The method is logical, such that parameter changes made by the user have the desired effect on the visualization.

The core idea comes from observing experts exploring volume data. When faced with a new data set they often start by sweeping the subranges of the intensity domain, so that they see what each part con- 


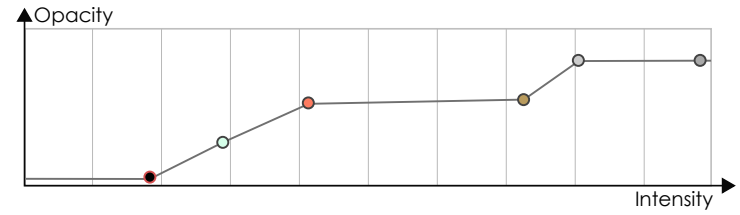

(a) Intensity-based transfer function. Without displaying any derived attributes there is no information about what effect the primitives (points) will have in the final rendering.

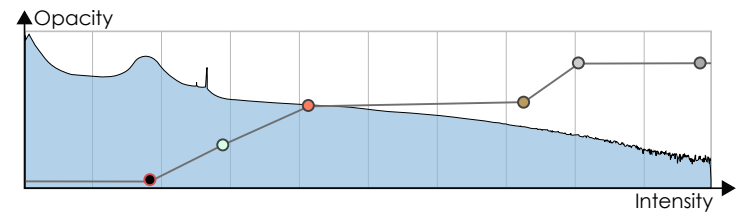

(b) Intensity-based transfer function with histogram. Adding a histogram (blue surface) gives some indication on where to guide the attention but it does not provide hints on the correspondence between values and the final rendering unless the user is very familiar with the data.

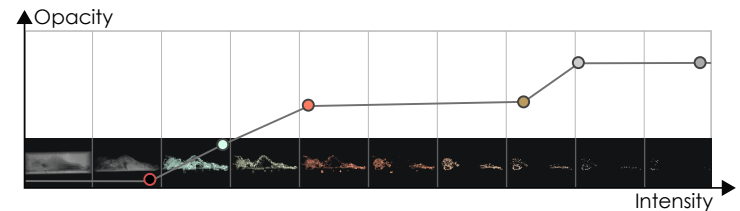

(c) Intensity-based transfer function with preview images. Displaying previews enables the user to make the connection between the data values and the final rendering.

Fig. 2: Comparison between different augmentations for transfer function editing. (a) and (b) demonstrate the difficulty in predicting the correspondence between a data value and the actual rendering. (c) by displaying a gallery it becomes possible to overcome the lack of spatial coupling and predict the effects of changing a range of data values.

tains. They then start constructing the visualization by adjusting the visualization parameters of each subrange that was found interesting. The base of our approach therefore heavily relies on interacting with these subranges and combining them with the design gallery method to gain intuitive and logical control. An overview the whole data set is provided by splitting the domain into equal subranges and providing a preview of each. By using such a simple method as dividing the attribute domain into equal parts, we gain an unbiased way of showing all the content within the data set as well as an easily explained and understood method. At this stage we let the user determine what should be emphasized through interaction. The user selects the ranges that are found interesting, which ensures that the following parameter changes are made locally in the intensity domain. Furthermore, to control how fine grained the changes can be, the user can zoom in and out in the data domain, thereby controlling the size of the subrange that can be edited. This enables the user to make changes to small or large data ranges in the intensity domain.

In essence, we couple the intensity domain with the spatial domain, and put emphasis on empowering the user with fluent interaction. Thus, unlike much of the previous work involving the transfer function for volume data, the focus of this work is on ease of use and interaction instead of improving the data classification.

\section{Related Work}

The most common way of exploring volume data is done by mapping visual parameters through the data domain. Expert users are commonly provided with a tool where the opacity, i.e. how visible the data values should be, and color can be adjusted for a given data range. An example of such a tool is given in Fig. 2(a), where the points can be moved and assigned a color.

The transfer function (TF) was first introduced by Drebin et al [7]

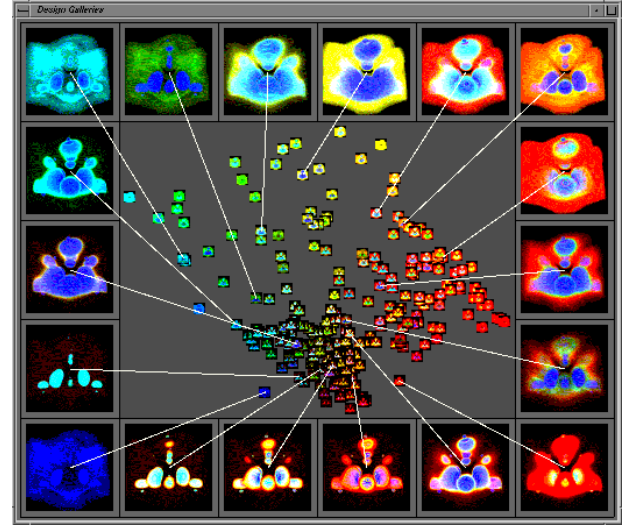

Fig. 3: The design gallery approach by Marks et al. [22] applied to a scalar field representing the electron density of a protein. Their work focus on changing all parameters at once while we allow the user to focus on one parameter at a time.

and their interfaces have been an ongoing research topic since then. Constructing the TF can be done using a set of components, defined by parametric functions, as suggested by Castro et al. [3]. König and Gröller [16] proposed a user interface for editing components defined by trapezoids and ramps, where previews help the user to get an overview. Pfister et al. [24] compared different image-centric and data-centric approaches. Rezk-Salama et al. [29] integrated domainspecific knowledge into the transfer function interface to make it more intuitive to novice users. More recent work by $\mathrm{Wu}$ and $\mathrm{Qu}$ [33] enables editing and combining images rendered by different TFs.

One of the fundamental problems with the common approach given in Fig. 2(a) is that it does not contain any information about the underlying data. To remedy this problem it is possible to display aggregated information in the data domain. Examples of this are the density distribution (histogram), local density distributions $[18,20]$ and isosurface similarity [1]. These approaches help the user to identify important regions, but do not provide spatial coupling. The spatial coherency between material types for transfer functions has been explored in several works $[17,19,30]$. The methods rely on local neighborhood analysis, but objects can also be described at a higher abstraction level, rather than local neighborhood analysis. Correa et al. [4] used the estimated object size and Prassni et al. [26] the object shape for this purpose. Sketch-based interaction for transfer function generation [11] and design [10,31] has also been explored, providing an interesting method of directly designing the TF onto the rendering. However, complex data with many varying spatial features increases the interaction complexity of these methods. Correa et al. [5, 6] introduced a visibility-driven approach to transfer function design. More recent work has focused on more advanced transfer function exploration utilizing hierarchical trees [13], model-based interface generation [32] or exploring an entropy map from a feature-clustering in high-dimensional space [34].

Several works have investigated how to semi-automatically design TFs. He et al. [12] use genetic algorithms to define good transfer functions for a given data set. The user can select a transfer function based on thumbnail renderings of the generated transfer functions. Kindlmann and Durkin [14] introduce a method which uses the data values as well as the first and second directional derivatives for generating a transfer function semi-automatically. Kniss et al. [15] extended that work with widgets and dual-domain operations. Marks et al. [22] propose the design gallery methodology which finds input parameters that produce perceptually different images and then displays them using an arrangement adapted to a specific application. Their tool for exploring volume data displays thumbnails in the center surrounded by an image gallery created by the user (Fig. 3). The input parameters for a given viewpoint and data set are found using an iterative approach that maximizes the nearest-neighbor distance in YUV color space of eight 
manually selected pixels. Once the transfer function parameters have been found, the layout of the thumbnails is determined using a distance preserving projection of the Euclidean YUV color space distance onto a two dimensional space. The user can pan and zoom the center view or select a thumbnail to enlarge it and put it into the gallery. Prauchner et al. [27] instead suggested a two-level approach that first displays a gallery emphasizing boundaries between homogeneous regions and then allows the user to change the color of the selected gallery preview image. Pinto and Freitas [25] expanded the two-level approach by enabling semi-automatic exploration in the first step, generating similar TFs based on the selected preview image, and adding more flexibility to edit the transfer function in the second step. Our approach differs in several aspects. While these methods focus on using a predetermined metric of what is important and changing all the parameters at once, we let the user decide what is important by changing one parameter at a time and instead rely on dynamically generating new previews as parameters change. This enables us to use a different layout, which requires less space, and reflects parameter changes, such as moving the viewpoint, without a substantial computational overhead.

There has also been work focusing volume exploration beyond the use of mouse and keyboard. Gallo et al. [9, 8] use a Wiimote, the controller for Nintendo's Wii gaming console, to move the camera and crop a volume with the goal of providing a more natural way to interact with volume data. Lundström et al. [21] presented a multi-touch system focusing on collaboration while exploring medical volume data. Mobile devices were evaluated by Butson et al. [2] to visualize and select deep brain stimulation parameters. Here, parameters similar to the standard practice could be selected in minutes instead of hours. A survey of volume exploration methods and transfer function designs can be found in the book by Preim and Botha [28].

To conclude, we believe that the gallery method is an useful and intuitive way for novice users to change the transfer function. However, since the previous gallery inspired methods only allow the user to choose from automatically generated transfer functions, rather than having an interface to control and explore transfer functions, full exploration into the data set cannot be gained from these approaches. The interaction for interfaces with full flexibility has mostly been centered around users familiar with the concepts around transfer functions and not to novice users.

\section{Application Domain}

The interest for interactive volume data visualization in museums and science centers is rapidly increasing, both due to digitization of artifacts, and due to advances in touch device technology, which has been shown to provide an intuitive way of interacting with the camera to explore volume data [21]. The museums and science centers goal is often to increase the dwell time in a gallery, i.e. the time spent in a gallery. Visitors to museums and science centers put a completely different demand on volume data exploration than domain specific users, who traditionally have been the target for volume data exploration tools. The main way of interacting with volume data in museums and science centers is today done by selecting from a set of presets that have been designed by an expert. Our goal is to make it possible for the user to further explore the data by changing opacity and color parameters.

Visitors typically only stay for a short while on each exhibit and may even pass by it if it does not look intriguing. When deciding to stay and explore it is essential that the user understand and see what the interactions result in. Otherwise, they will lose interest and miss the opportunity to discover, and learn about the data. Explanations on how to perform the interactions are usually embedded into the application, using for instance, overlay help symbols and text, which can be displayed when the visitor approaches or presses a help button. Preferably, additional instructions should not be needed to understand how to use the application. There are sometimes guided tours, where more time is spent on an exhibit and it is therefore even more desirable to explore the data beyond the existing presets.

A set of requirements was formed from the experience of the application domain:
R1 A new user should be able to perform interactions with little explanation.

R2 An intriguing design should attract the user to interact with the application.

R3 Interactions should give feedback such that the user quickly can understand the effect.

R4 The application should be responsive for smooth interaction.

R5 It should be engaging and fun to use.

R6 Users should be able to explore data beyond presets.

\section{Method}

Our approach to address the lack of spatial coupling, illustrated in Fig. 2(a)-(b), is to dynamically generate and display previews of subranges of the data. Each preview can be seen as a window into the data domain and navigating this domain is done through panning and zooming the windows, effectively changing where the subranges should be placed and how large they should be. As can be seen in Fig. 2(c), the spatial coupling with the data range becomes direct when it is possible to see in the preview image where the data values are spatially located and are thus affected by a parameter change. However, moving primitives around to change parameters is often not familiar to a novice user and, in addition to this, understanding that moving a primitive up and down corresponds to changing the opacity requires the user to interact with the primitive in the first place.

We instead propose to completely hide the traditional primitives and let the user interact through the automatically generated gallery that spans the entire data domain. While other approaches have generated galleries, they have often done so based on finding a set of representative images in the parameter space as a precomputation step, which the user then can navigate in $[12,22]$. This kind of navigation is problematic since it spans a high-dimensional space, which to the author's knowledge is yet to be shown how to navigate in an intuitive manner, and also relies on knowing beforehand what the user is interested in.

Our approach is conceptually simple; the gallery only consider one dimension at the time, fixing the other parameters, and relies on dynamically recomputing the gallery when changing parameters such as camera or color. No precomputation is therefore needed since we rely on progressively generating previews.

Changing the parameters for a subrange can be done by selecting a gallery image, which fixes the intensity domain parameter, and then exploring the opacity through a new gallery that spans the opacity for the given data range, see Fig. 1 (right). The effect of changing the opacity therefore becomes apparent without needing to edit the parameter beforehand. The color space requires three dimensions to be explored. This presents a challenge for the interaction on the screen, where only two dimensions are available, and which is usually solved by displaying two dimensions against each other and fixing the third. We investigated how to change the color with less interaction and came up with a solution that reduced the interaction to two dimensions without restricting the available color space too much.

In the following, we explain how to improve editing opacity and color. We then describe the design process followed by the evaluation of the user study and a discussion of the limitations.

\subsection{Nonlinear Opacity Editing}

A straightforward implementation of a transfer function maps the opacity linearly from zero to one. However, a linear change of the opacity will create a nonlinear change in the rendered image due to the exponential behavior of the transmittance term in the volume rendering equation [23].

In order to produce a more informative gallery we propose to revert this nonlinearity, such that an opacity change behaves in a more linear fashion for the user. We will first derive the mapping by looking at the transmittance before discussing how to apply it in the application. The transmittance between two points $s_{1}$ and $s_{2}$ is given by:

$$
T\left(s_{1}, s_{2}\right)=e^{-\int_{s_{1}}^{s_{2}} \sigma_{t}(s) d s}
$$




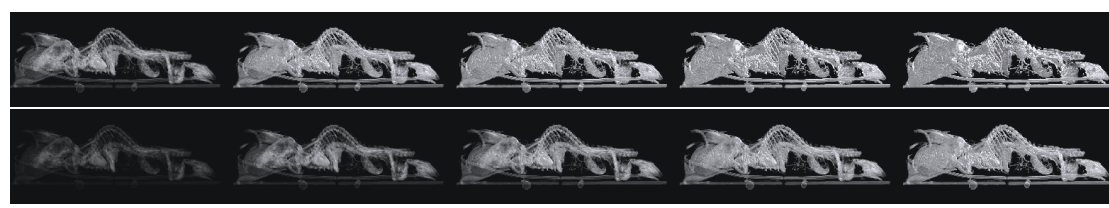

(a) Displaying skeleton and internal organs.

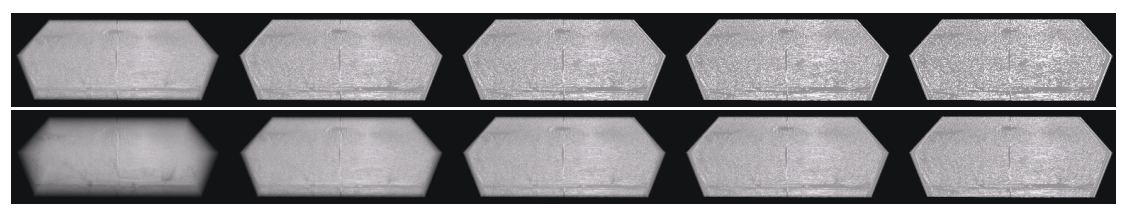

(b) Displaying air.

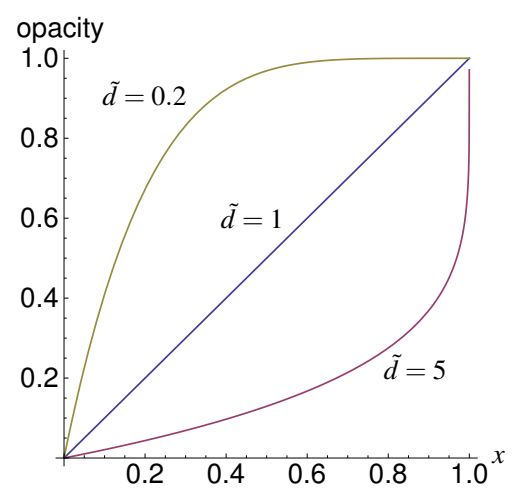

(c)

Fig. 4: Preview renderings of a micro CT-scan of a mouse for different opacity values. The top rows in (a) and (b) have been created using a linear opacity, ranging from 0.1 to 0.9 in steps of 0.2 . The bottom rows have been created by mapping the same opacities through Equation 4 using $\tilde{d}=5.0$. The bottom rows, which uses the nonlinear mapping function, produces a much better overview of the data and also allows for a more intuitive and easy control of small opacity values. (c) Mapping function for different values of $\tilde{d}$ in Equation 4.

where $\sigma_{t}(s)$ is the extinction coefficient at location $s$. Assuming a constant extinction coefficient $\sigma_{c}$, which will be used to create the preview for an intensity range during the opacity editing, and $d=s_{2}-$ $s_{1}$, we end up with the nonlinear expression:

$$
T\left(s_{1}, s_{2}\right)=e^{-\sigma_{c} d} .
$$

In order to allow the user to specify the extinction using a normalized value $x \in\left[\begin{array}{ll}0 & 1\end{array}\right]$, we apply a mapping function that utilizes a logarithmic function to revert the nonlinearity:

$$
\sigma_{c}=f_{\text {extinction }}(x)=\left\{\begin{array}{cl}
-\ln (1-x) \frac{1}{\tilde{d}} & 0<x<1 \\
1 & x=1
\end{array},\right.
$$

where $\tilde{d}$ is a normalization factor that enables us to retrieve a linear result for rays that are not of length one, see Fig. 4(c). The normalization factor is needed since we cannot change the length of the rays passing through the volume. For rays longer than the specified parameter $(\tilde{d}<d)$, the effect will be that the transmittance will be pushed down, while it will be pushed up for shorter rays $(\tilde{d}>d)$. For the sake of completeness, we also provide the mapping function for opacity:

$$
f_{\text {opacity }}(x)=1-(1-x)^{\frac{1}{d}} \text {. }
$$

It is now up to the application to decide the normalization factor $\tilde{d}$. Dynamically setting the parameter per data range can produce an accurate estimate $(\tilde{d} \approx d)$, which guarantees that the material is visible in the first preview and fully opaque in the last one. However, we found that using a global value of 5.0 also produces acceptable results unless thin materials are examined. For thin materials, where $d \ll \tilde{d}$, it follows from the derivation that the opacity is not adjusted enough and they may therefore be hard to see. The effect of using linear versus nonlinear opacity editing can be seen in Fig. 4. This simple adjustment increases the value of the previews generated for the opacity and we believe that this technique would be beneficial in all transfer function editors, such that small changes for low opacity would not result in a large visual change.
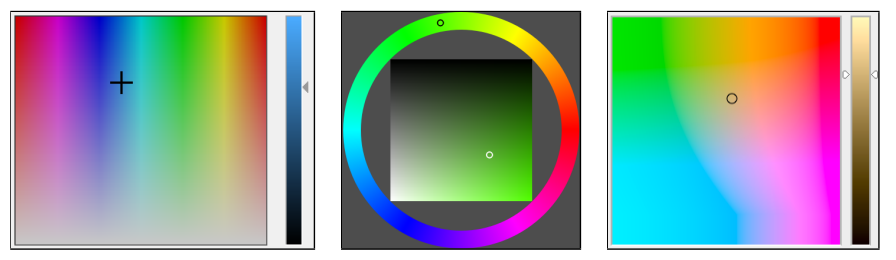

Fig. 5: Different color picking approaches: HSV color picker, HSV color wheel, and $\mathrm{L}^{*} \mathrm{a}^{*} \mathrm{~b}^{*}$ color picker (left to right).

\subsection{Color Editing}

When dealing with colors, a color picker is typically used for the interactive selection and adjustment of a color. There exists a wide range of different interfaces for picking a color, including sliders and spin boxes, but in most cases a graphical representation of the color space is used. All these interfaces have in common that there is no single-click solution for choosing a color, except for color presets or palettes. As the color spaces have three or more dimensions, a dimensionality reduction is necessary before mapping the colors onto the screen. Color pickers therefore usually combine two dimensions of the color space resulting in a filled rectangle while the third axis is either considered to be independent or used to change the appearance of the rectangle. Variations of this concept are depicted in Fig. 5. To choose a color, the user has to select a point in the rectangle and a second point in the vertical bar or color wheel, respectively. Depending on the predefined axes, the color choice with a single click is rather limited.

To overcome this restriction and to simplify the process of color selection, we decided to utilize the concept of a color sphere onto which all three dimensions of the color space are mapped. Fig. 6 shows the concept of the color sphere for the HSV color space. The interaction is constrained to a disk where the radial angle determines the hue. Saturation and color value are linked and changed simultaneously. The disk itself is divided into two parts, an inner disk and an outer ring. Within the inner region the value is fixed while saturation and hue can be adjusted. In the annulus, the saturation is fixed and the value varies. These two constraints prevent, however, to choose desaturated colors with a varying value, for example different gray values. Yet, the color sphere is able to provide a larger variety of colors in a 2D area than the color pickers mentioned above. Although not all colors are represented in this color sphere, this fact is outweighed by the advantage of being able to select a color with only one interaction.

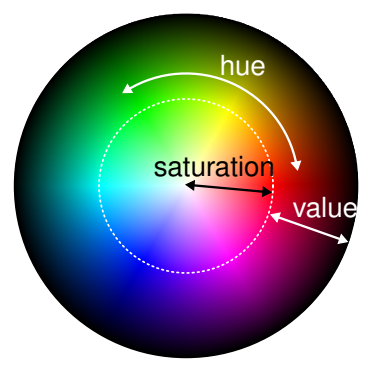

Fig. 6: Color sphere picker used in our study requiring only one interaction to select a color. Saturation is changed in the inner part of the sphere with constant color value. In the outer region the colors are fully saturated while the value can be adjusted. 


\section{The Design Process}

Sketches were initially used to investigate what kind of layout and workflow would be intuitive to use. In a second step, a series of images were produced by rendering a set of isosurfaces and printing thumbnails on a piece of paper, which could be rearranged to simulate interactions. This enabled us to quickly explore alternatives that affected requirements R1 (intuitiveness), R2 (intriguing design), R5 (engaging) and R6 (exploration). Different methods to interact with the previews and editing the parameters they represented were brainstormed. The brainstorming resulted in, for instance, a drag and drop area as can be seen in Fig. 7. The first prototype used an isosurface to represent each subrange of the data. However, these were found to be incapable of representing the data accurately enough, which led to the use of volume rendering and trapezoidal primitives covering the data range.

Once the prototype could be used, it became clear that having a large set of previews was not optimal. Partly because splitting an intensity range is equivalent to zooming in on the data domain, which means that zoom and pan can be used instead, but also because it could become difficult to see, tedious to edit large ranges and it produced more distractions (R2). After letting a few users with expert knowledge try the prototype it was determined that roughly five images were sufficiently flexible and did not distract too much attention from the other elements. In comparison with the design in Marks et al. [22], our approach is able to use much fewer previews due to the support for interaction and the use of dynamic galleries. It was also determined that clicking on an image to edit it would be preferable to dragging and dropping, since it would both preserve more space and involve less interaction, even though dragging and dropping could be more playful (R1 versus R5).

A range slider was added below the gallery in order to give an overview, possibility to see the current location in data space, and feedback on zoom/pan interaction (R3). The range slider was decorated with indicators, displaying the selected color of each subrange in order to connect the slider with the previews, see Fig. 8. The indicators outside of the range slider were darkened to emphasize that they were not part of the displayed gallery. Similarly, the same indicators were shown beneath each gallery image, acting like a zoomed in view of the area in the range slider. Pressing an indicator would center the gallery on the selected data range and therefore also serve as possible navigation. The layout of the application was at this point fixed to a main visualization area, a dynamic gallery area, and an overview area as seen in Fig. 9(a). The parameters of all areas were kept synchronized, such that changing the camera in the visualization area is reflected in the gallery and changing color or opacity in the gallery is reflected in the main visualization area.

\subsection{Pre-study}

Having the layout set, we turned our attention to the interaction (R1, R3, R4) and how to edit different parameters before performing the user study. We formed the questions given below, discussed options, and then performed a pre-study with four novice users, two women and two men, ages 8, 27, 39, and 74 years old. We will first discuss the questions we wanted answered, the options we considered, and then the result of the pre-study.

\section{Guided or direct navigation to opacity and color editing?}

We previously discussed that clicking on an image would make it possible to change its corresponding parameters. We wanted to know if a guided approach should be used, where the user is first editing the opacity, then the color, and then going back to the main view, or if it should be possible to go directly to editing color and opacity. Using the guided approach it would not be necessary to introduce more elements in the data range gallery but it could be cumbersome if the user only wants to edit one of the parameters, while the direct approach would require additional information on how to start editing.

\section{What should happen when the user translates the gallery?}

Two different types of interaction were discussed. The first moves the camera and fixes the data domain. This would correspond to walking

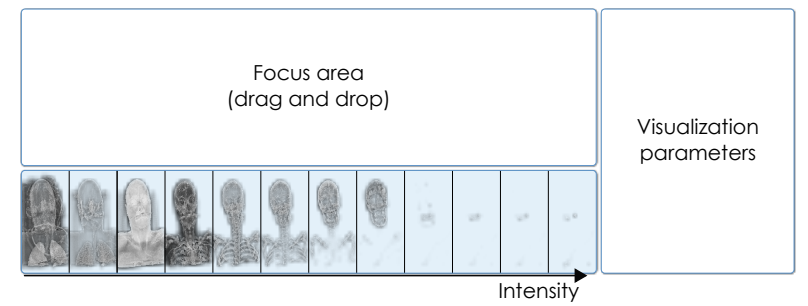

Fig. 7: One of the first sketches for the interaction included a drag and drop area as well as proof of concept for the gallery.

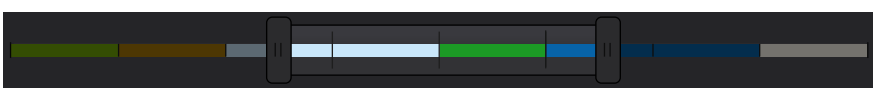

Fig. 8: Preliminary designs included indicators (colored rectangles) on the range slider, displaying the selected color of a subrange, in order to connect the data domain with the gallery.

on a shopping street while looking into the windows displaying the objects inside. The second involves fixing the camera looking through the windows at the data domain and translating the data domain. This would correspond to standing still on a sidewalk, looking into a window, and letting the objects behind the window move.

3. Should the data range that is not visible in the gallery be filtered out in the final rendering?

The data range displayed in the gallery is determined by the translation and zooming in the data space, the green area inside the range slider in Fig. 9(c). The advantage of displaying the whole data range is that it is possible to compare all features with the ones in the gallery. The disadvantage is that it becomes difficult to focus on a specific feature, especially in the frequently occurring case when it is enclosed by another feature.

The participants in the pre-study explored three different data sets, one for each configuration. There was no time limit and they were free to explore the data sets more if they desired and asked to "think out loud" describing what they did, why they did it, and what they would like to do. They did not get any compensation for participating in the study. We varied the parameters discussed in the questions above and observed how they interacted. After they had completed the tasks, each participant was asked which type of interaction they preferred or if they did not notice a difference.

For the first question we observed that the guided editing resulted in a tedious work flow since the users were not structured in their changes, which resulted in skipping either changing the opacity or the color. For the color editing, we displayed the color in the background of the gallery to help predict intermediate colors between the previews. However, since the gallery pictures had the same color as the background they did not add much additional information. Despite little additional information added from the gallery previews, they did not have much problems predicting the color that would be produced in the final rendering. This indicates that choosing a color is a task that does not require a gallery to intuitively understand the effect of or get the desired result. It was therefore decided to merge the two steps into one, similar to the standard transfer function, where a gallery for the opacity is displayed together with a color picker. This also removed the need to have additional information in the main data exploration view to edit opacity or color (R1).

For the second question we did not observe much difference in how they interacted and the users themselves had not noticed any difference when asked which one they preferred. It should be noted that some of the users also did not use the zoom and pan functionality while exploring. Nevertheless, from this we conclude that the type of translation does not have a major impact on the user experience and chose to use the approach where the camera is moved and the data domain is fixed due to its similarity to browsing an image gallery. 


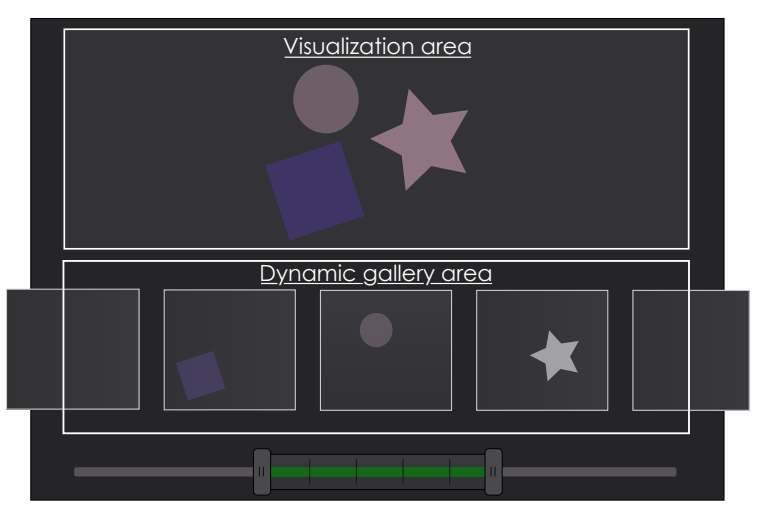

(a) After a number of iterations and tests with both expert and novice users the layout was finalized. The main elements are a visualization area, containing the final rendering, a gallery area, containing the dynamically generated previews, and a range slider area, providing an overview of the data range.

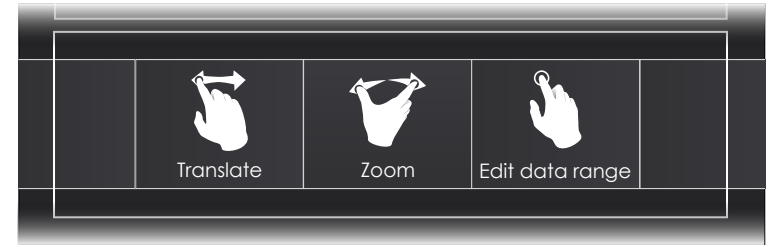

(b) Available touch-based interactions in the dynamic gallery area. Boxes represent gallery images, which act like windows into a data range.

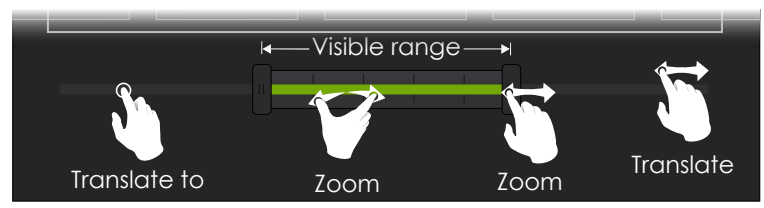

(c) Touch-based interactions in the range slider area.

Fig. 9: Overview on the finalized application. (a) final layout. (b) and (c) available touch interactions for both gallery and range slider areas.

For the third question we made two interesting observations. First, seeing features in the final rendering that were not displayed in the gallery was confusing to the user. Second, while editing a feature that is contained in another feature, for instance, the coins covered by the shell in the right column in Fig. 11, the user did not get any feedback on the changes which they thought was very ambiguous. In this case, an expert user can often make the connection that it is hidden inside, but a novice user does not have previous experience of this. We therefore only display the visible data range in the final rendering and suppress the opacity of everything else by $90 \%$ (R3) while editing. The disadvantage of reducing the opacity while editing is that the result will look different from the final visualization. It will be investigated in the user study if this disturbs the user.

A final remark that we received in the pre-study was that it was difficult to make the connection between the indicators on the range slider and the gallery images. The indicators on the range slider instead took focus and cluttered the interface, which caused us to remove them and use a single color inside the range slider. The indicators below each gallery were kept, since they were easier to connect to the gallery.

\subsection{Interaction Schemes}

To enable intuitive data exploration with our application, the interactions should be easy to understand and perform (R1), in particular for novice users. We implemented functionality for a set of common touch gestures known from mobile devices, pinch, drag and pan.

\subsubsection{Touch Gestures}

Navigation: The possible interactions for navigation are depicted in Fig. 9. They are divided into touch gestures for the dynamic gallery and gestures for the range slider area.

The gallery area allows the user to edit individual data ranges by touching them. The drag gesture pans the gallery windows left and right while the pinch gesture adjusts the zoom.

The range slider area provides an overview and shows the currently visible data range, which means that the touch interactions are focused on panning and zooming. The user can zoom in or out, i.e. modifying the size of the data range, by either dragging the left or right handle or by using the pinch gesture. Dragging the handles results in enlarging or shrinking the visible data range with the other end being fixed. In contrast, the pinch gesture zooms in on the point lying in the middle between the two fingers. A single touch in the slider area positions the data range window under the finger while dragging translates the slider relative to its position.

Editing Mode: When the user touches a gallery window, the user interface switches to the editing mode where the color and opacity of the windowed data ranges can be adjusted (Fig. 1, right). In this mode, the lower left part is filled with preview images illustrating the effect of different opacities. We apply the nonlinear opacity model with increasing opacity from left to right as depicted in Fig. 4(b). The color picker is positioned in the lower right corner.

If the currently selected window contains only one data range, the user can change opacity or color by dragging or touching the desired value with immediate feedback. By choosing a new color, the preview images are updated from right to left to reflect the change. This order was chosen as to ensure a coherent context since the user is focusing on the color picker and the rightmost preview is most likely to appear in the user's peripheral vision (R3, R4).

The interactions change slightly for multiple selected data ranges. A single touch now assigns the chosen opacity or color to all data ranges. The drag interaction, however, ensures a relative change, i.e. the distance between the different ranges remains constant. If both opacity and color are equal for all data ranges, they are merged into a single range.

\subsubsection{Mouse Interaction}

The user interface has mainly been designed with touch interaction in mind but is also functional when using a mouse. Single touch gestures and dragging are mapped to the left mouse button. The pinch gesture for zooming is replaced with scrolling the mouse wheel and considering the current mouse position as the central point.

\section{IMPLEMENTATION}

The previews placed in the dynamic gallery are generated and edited using trapezoids in the standard transfer function, which in fact enables support for all existing code that uses the intensity-based transfer functions. The height of the trapezoid determines the opacity and a constant color is applied to the entire subrange. When compared with adding individual points to a standard transfer function, this trade-off simplifies interaction while still retaining large exploration possibilities. High frequency changes between neighboring trapezoids, which can be visually disturbing, are avoided by removing the transition to zero between them. The overall effect is a linear transition between close subranges, while isolated data ranges will be represented by trapezoids. An example of this is shown in Fig. 11(b).

While generating a single preview image is usually fast enough to not noticeably affect the interaction, generating multiple previews per frame may slow down the system. Therefore, in order to meet requirement R4, a progressive scheme was implemented. The progressive scheme makes use of a render queue containing a prioritized list of gallery images to update. A new item from the render queue is processed if the estimated time to render it is less than two milliseconds. The time estimate to render is based on the previous item in the queue, but is always initialized to zero such that at least one item can be processed per iteration. 

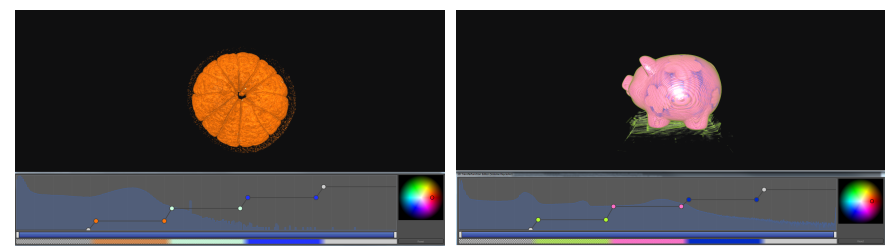

(a) View for standard transfer function with the histogram (log-scale) in the background.
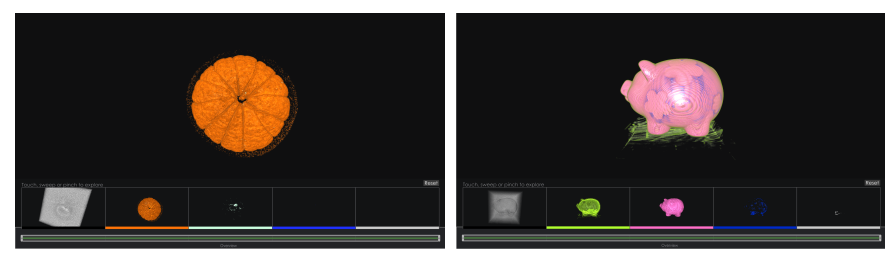

(b) View for our dynamic gallery method.

Fig. 10: The initial study setup shown to the participants for the orange (left) and the piggy bank (right) data sets.

The prioritization in the render queue is dependent on the type of parameter that has changed in order to further improve the responsiveness of the application. For camera interaction the update start in the center of the gallery and works its way outward. The same order is applied when zooming and panning in the dynamic gallery as well as when initiating the opacity and color editing. However, when the user changes the color we update the gallery image closest to the color dialog first, followed by the next closest image and so on. Changing the opacity does not require the gallery to be updated.

The items in the queue that are not able to complete are showing the closest data range previously rendered. For this purpose, we maintain a cache of previously rendered gallery images which makes it likely to find a similar data range and thereby a similar image. This gives a smoother experience, especially when panning and zooming in the dynamic gallery, than showing empty previews since they are often similar to each other. The progressive approach also enables large data sets to be used without impacting the interaction noticeably, examples of the interaction behavior are shown in the supplementary material.

The implementation itself is written in $\mathrm{C}++$ and uses a mixture of OpenGL and OpenCL for the graphics and rendering. The same renderer is used for both the preview area and the main visualization area in order to make sure that the user get as good estimate as possible of what the final rendering will look like.

\section{USER STUdY}

The applicability of the method was evaluated by performing a user study at a science center. The idea was to select users that were as close to the target group as possible and perform the study in the environment that they would use the system in. The target audience of the science center is the general public, ages from six years and up, with no specific prior experience in computer usage.

The goal of the study was to find out if the spatial coupling with the data domain makes it more intuitive to explore the data, if they enjoyed the exploration and if they thought that the application invited them to interact. In order to investigate if the users improve the spatial coupling with a dynamic gallery, and to see if they prefer the method against the most frequently used volume exploration tool, we made a comparison to the standard intensity-based transfer function, which we adapted for touch screen usage. While the standard transfer function could have been left unadapted, we wanted to investigate the spatial coupling, and not confuse the user with additional settings or tedious touch interaction. The adaptation is further described in Section 7.4. A predominantly qualitative study was performed, where each task was followed up by a brief quantitative questionnaire. After performing all of the tasks, they were also asked which of the two methods they preferred to use. The study took place before Easter, so in order to attract the participants to take part, and use a theme fitting the season, the idea was to arrange a virtual Easter egg hunt.

\subsection{Participant Profile}

Sixteen participants took part in the study, six women and ten men aged between six and forty years with an average age of twenty six. The participants in the study largely reflect the target audience of the science center, where the majority are children with accompanying parents. None of the participants had previous experience of exploring volume data using transfer functions, but some had used the exhibit in the science center displaying volume data, which meant that they were a bit familiar with the camera movements. All of the participants had used touch devices before, fifteen specified that they used it on a daily basis while one specified sometimes (choices included never, seldom, sometimes, daily). They received no compensation for taking part in the study.

\subsection{Data Generation and Setup}

The two data sets that were selected were a piggy bank and an orange, see Fig. 10 for the initial setup for each method. Scans of humans were avoided for ethical reasons and we instead opted for data sets that would be more familiar to the users. The orange data set size is $256 \times 256 \times 64$ voxels, where an egg, 48 voxels wide and 40 voxels high, was centered inside the orange at voxel coordinate $(88,156$, 26) using a normalized intensity between $\left[\begin{array}{ll}0 & 1\end{array}\right]$ of 0.46 . In order to reveal the egg inside the orange they would need to either decrease the opacity of the fruit meat, or use the range slider to filter out the egg. The piggy bank data set is size is $512 \times 512 \times 134$ voxels, where an egg, 48 voxels wide and 40 voxels high, was centered inside the piggy bank at voxel coordinate $(185,313,54)$ using a normalized intensity between $\left[\begin{array}{ll}0 & 1\end{array}\right]$ of 0.16 . Revealing the egg in the piggy bank was more challenging as it was hidden in the lower ranges of the data close to air and concealed within the case. The user would either need to zoom in on the egg (possibly using the range slider) to filter out the surrounding material or, in the case of the standard transfer function, first decrease the opacity of the case in order to find it. The egg was also not visible in the gallery from the initial setup in the piggy bank. Possible solutions to the finding the egg in each data set are shown in Fig. 11. The hardware used in the study was a 27 -inch touch screen with $1920 \times 1080$ resolution connected to a computer with an NVIDIA 670 graphics card.

\subsection{Task and Procedure}

A virtual egg was hidden in a data set at a location, and with an intensity value, that was intended to be somewhat challenging. The task was to find and reveal the Easter egg and assign it a color of their preference. This would enable us to observe how they used the two different methods for exploring the data, and if they understood the link between opacity, color and data range.

Each participant performed the tasks individually, although, since the study was performed at one of the exhibition floors, it cannot be ruled out that a participant had seen someone else interacting before. When a group of people agreed to participate, the ones not performing the study were kindly asked to enjoy the other parts of the science center. A written instruction of the study was first presented to the participant and, after agreeing to participate, they were given a quick demonstration of the interaction of each method, lasting in total roughly one minute. The participants were informed that they could interrupt at any time and that, since we were not interested in how fast they were performing, they were free to investigate other interesting features in the data if they desired and should not feel any pressure. The study began with a set of questions about the demographic background, age, gender, previous usage of touch devices and previous experience of volume visualization. The participants were asked to "think out loud", describing what they did, why they did it, and what they would like to do. Each participant tried to find the egg once with each method, where the order and combination of the method and the data set was randomly chosen. They were only assisted during the task if they explicitly asked for help. After using each method they completed a short 

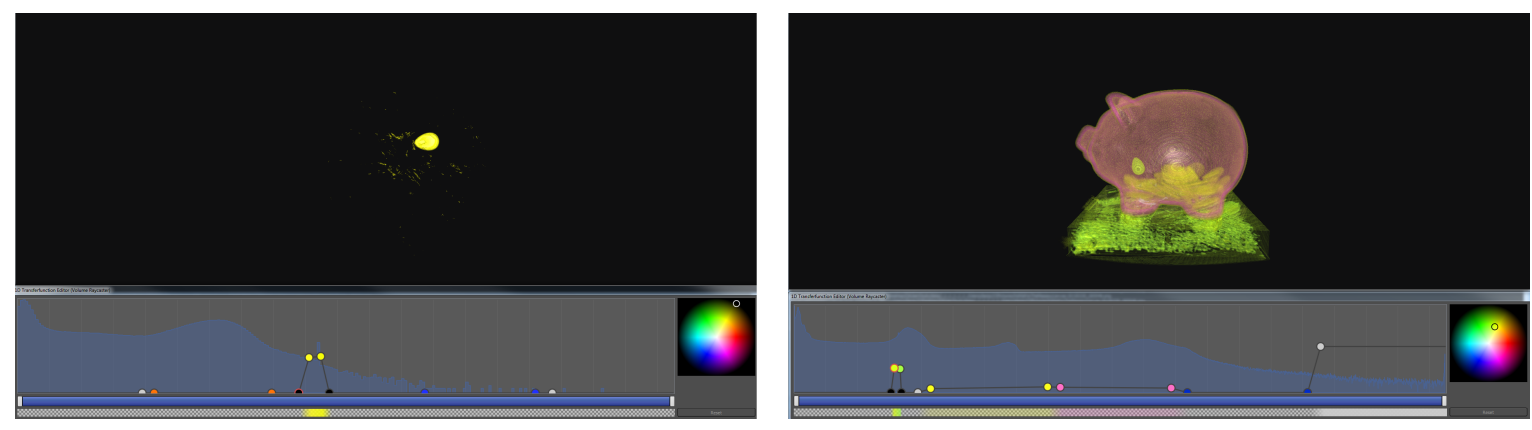

(a) One possible way of adjusting the transfer function to reveal the Easter egg. In the piggy bank transfer function setting (right) one can see the ambiguity of the yellow points, which of them correspond to the egg in the final rendering?
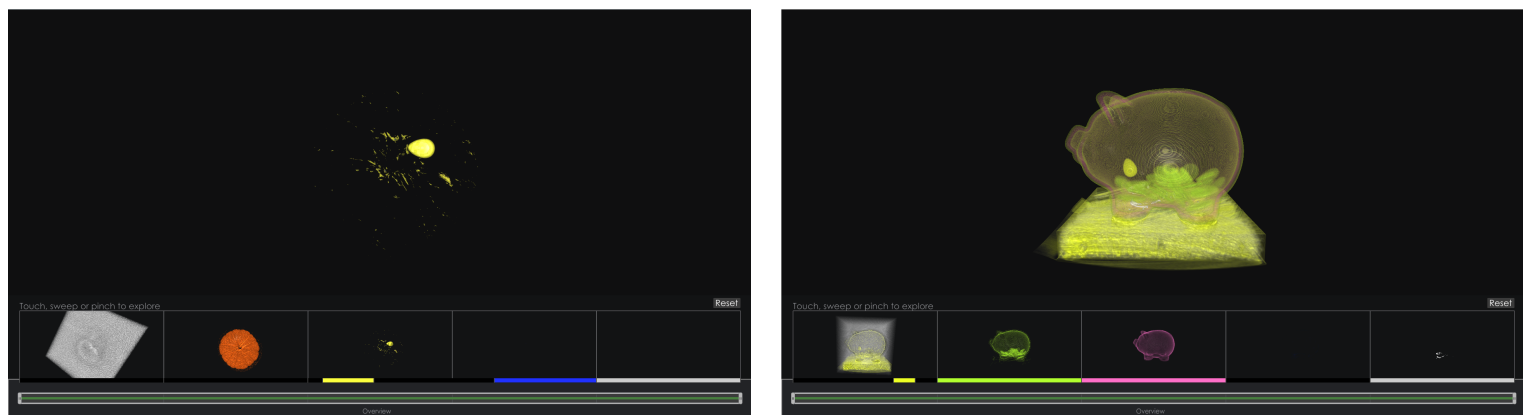

(b) One possible solution using our dynamic gallery method. Extracting the egg in the piggy bank data set (right) required the participants to zoom in the data space since it was otherwise hidden by the surrounding material in the previews.

Fig. 11: The goal of the user task is to find a hidden Easter egg and colorize it: orange data set (left) and piggy bank data sets (right).

subjective satisfaction questionnaire. Each question had a 5-point rating scale: Strongly unfavorable (1), Unfavorable (2), Unsure (3), Favorable (4), and Strongly favorable (5). The issues posed where:

- Easy to learn: Did it take long time to understand how it worked.

- Easy to use: Did they find it easy to use once familiarized with it.

- Fun to use: Whether they enjoyed using the tool.

- Inviting design: Did they like the look and think that it was engaging.

- Would try it in an exhibition: Whether they would walk past or start using the application in an exhibition.

After completing both tasks they were asked which method they preferred and also state why they preferred the chosen method. The tasks and questionnaire were intentionally kept short, lasting roughly ten minutes in total, to respect that they were visiting for other purposes.

\subsection{Adaption of The Standard Transfer Function}

The standard transfer function was simplified and adapted in order to work better for touch devices and the target audience. The color picker was replaced to the simpler version, see Section 4.2, which require less interaction. Furthermore, the sizes of the points and the range slider were increased to be easier to interact with when using touch devices. In terms of interaction, we made it possible to select and edit multiple points at the same time by dragging a selection box around the points See Fig. 12 for a reference of how the editor looked before and after.

\subsection{Results}

Out of the sixteen participants, three favored the standard transfer function and thirteen favored the dynamic gallery approach when asked afterward which one they preferred to use. The questionnaire indicates the same, reporting a favor toward the gallery approach as shown in Fig. 13. Most people thought that it was "fun" to explore volume data using both approaches, except for one child who was disappointed that he did not find the egg and one adult who was frustrated with the standard transfer function.
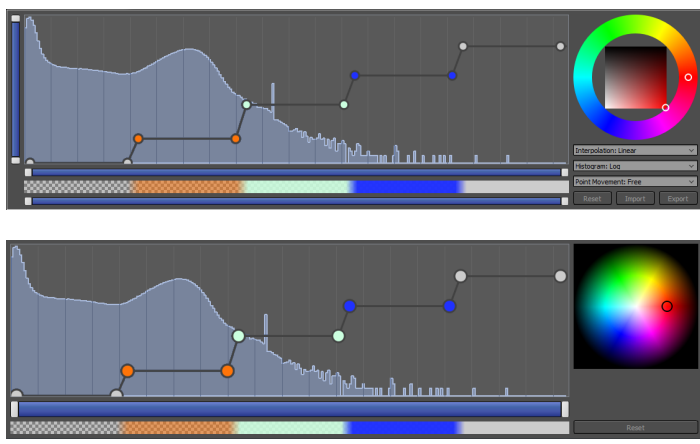

Fig. 12: The standard transfer function used by experts (top) was simplified and adapted for the user study (bottom).

For both of the methods, very few participants used the range slider and once doing so, they were surprised by what it did even though the functionality had been demonstrated before. We also observed that many participants pressed on the screen in the visualization area to see if they could edit the object, a functionality that both methods are missing.

\subsubsection{Standard Transfer Function}

When observing the usage of the standard transfer function, it became clear that it was difficult to understand that one first had to select a point in order to edit the color of it. Many of the participants tried to change color while not having a point selected and some changed the color without being aware of which point that was selected. Most of the participants grasped how to change color and opacity after experimenting a bit with the points. However, when making informed decisions, for instance trying to assign a color to the egg, it was necessary to make a clear connection between the color and/or opacity of the point and the egg (feature). Some of the participants managed to make the connection, but sometimes it was also complicated by the 


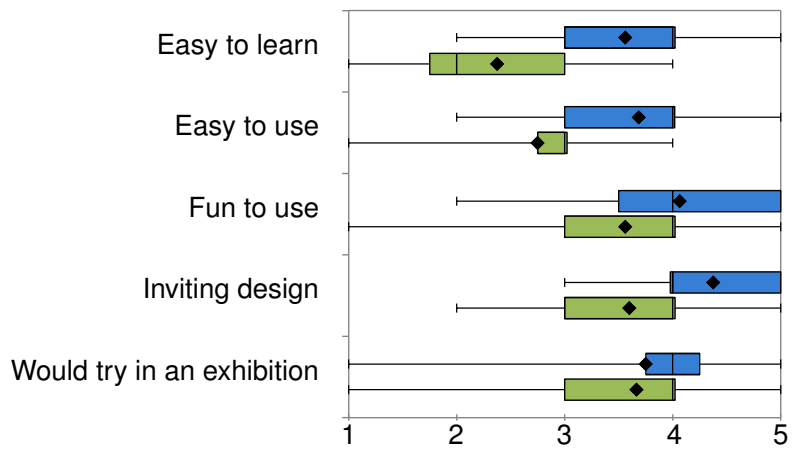

Fig. 13: The quantitative results from the user study questionnaire for our method (blue) and the standard transfer function editor adapted for touch devices (green). The 5-point rating ranges from strongly disagree (1) through unsure (3) to strongly agree (5). The whiskers stretch the full range of the answers while the boxes show the range from the first to the third quartiles, divided by the median. The black diamonds mark the mean values of the answers.

fact that two different features had the same color, see Fig. 11(a) for an example. Another difficult scenario that occurred was when a feature had a color that was interpolated between two different points, making it even more difficult to know what to change.

The advantage of being able to edit the whole data range using the same type of interaction became visible when the participants explored the piggy bank data set, where more people managed to find the hidden egg. This required a different type of interaction for the dynamic galleries. A severe drawback with the standard transfer function was that it did not provide any feedback on changes made to features occluded by other features. Thus, almost all participants changed opacity or color of points that did not have any effect on the final rendering. This was very unintuitive to the participants who expected some kind of change. The image at the bottom, containing an overview of the transfer function turned out to be more distracting than useful as some tried to interact with it without anything happening. The two comments received favoring the standard approach were "It was easier to use and understand for someone who has not used it before" and "Easier". One participant said that "it is challenging in the beginning, it becomes a little bit more like a game" about the standard transfer function after spending a bit of time familiarizing with the interface.

\subsubsection{Dynamic Galleries}

The usage pattern for the dynamic galleries differed from the standard transfer function in that the user did less experimenting and more conscious decisions on what to edit. The most prominent problem the participants had with the dynamic gallery method was the that they entered the mode for editing opacity and color, but did not know how to get back or forgot that the main view existed. These were also the participants who gave lower scores on how easy it was to use. A couple of participants pointed out the usage of the word "Done" to get back as one source of this problem. Compared to the standard transfer function, with the editor always being visible, it was harder for some users to remember how to start editing a gallery image.

In the pre-study it was determined that the opacity of everything except the data range currently being edited should be decreased such that the user got feedback when changing a parameter. This turned out to be essential for the novice users as they now always got feedback on the changes made. None of the participants reacted to the change in the rendering when they went back to the main view.

While the participants zoomed in the final rendering, it did not seem to be intuitive for them to start zooming in the gallery, as very few utilized the functionality. Comments favoring the gallery approach included "Better overview, clearer and easier to understand and use", "More clear. More fun", "Easier and more fun with pictures", and "It did not have so many things to keep track of." Fig. 14 illustrates a mockup of an improved design after taking user comments and experi-

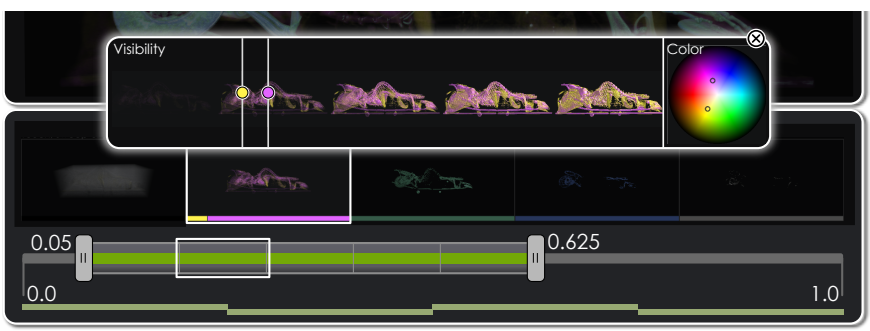

Fig. 14: New mockup design based on the findings in the user study. Opacity and color editing now preserves the context while highlighting the active gallery subrange. Labels are positioned next to the scale bar to make the selected data range appear more explicit and inviting for interactions.

ences into consideration. The changes incorporate a floating dialog for editing color and opacity besides a more interaction-friendly scale bar.

\section{LimitaTiONS}

Similar to most other transfer function interfaces solely based on the intensity domain it can be hard to detect and manipulate features that span a narrow intensity range. In this case it would be beneficial to provide a sub-division scheme that divides the domain according to features instead of a uniform scheme. The current implementation does not handle classifiers based on multiple attributes, such as intensitygradient [14]. These classifiers can, in many cases, provide a better separation of the features within the dataset but also require more interaction. A simple solution for two dimensions would be to introduce a second axis in the gallery area at the expense of smaller previews. Still, more work is needed to investigate how the interaction can be reduced and performed in a transparent manner for the user.

\section{Conclusions AND FUtURE Work}

In this work, we presented a novel interaction method for novice users based on dynamically generated galleries. The method was designed from the ground to work well with touch interfaces and techniques for interacting in the data domain were evaluated in an iterative design process. We investigated and presented ways of improving the galleries generated for opacity and how to decreasing the interaction necessary to change the color.

Finally, we performed a qualitative user study, where we investigated the impact of the spatial coupling between data domain and the spatial domain by comparing our method to a standard transfer function adapted to touch interfaces. The study showed that the participants made more conscious decisions on what to edit when presented with the spatial coupling and that they preferred to use our dynamic gallery method. The study also confirmed that novice users find it more intuitive when the opacity of everything else is reduced in order to provide feedback and reveal the edited feature.

Next steps include investigating ways of making it more apparent that the user can zoom in on the data domain using the galleries and edit color and opacity as well as decreasing the context switch when starting to edit. Methods for supporting editing through touch in the visualization area should also be incorporated. Interesting avenues for future work also include integrating new interaction devices and studying how the design should be adapted to smaller screens.

\section{ACKNOWLEDGMENTS}

The authors wish to thank Camilla Forsell for advice on the user study and the Visualization Center C, Norrköping, Sweden, for supporting the evaluation. This work was supported in part by the Swedish Research Council, VR grant 2011-5816, the Excellence Center at Linköping and Lund in Information Technology (ELLIIT), the Linnaeus Environment CADICS, and the Swedish e-Science Research Centre (SeRC). The presented concepts have been developed and evaluated using the Inviwo framework (www. inviwo.org). 


\section{REFERENCES}

[1] S. Bruckner and T. Möller. Isosurface similarity maps. Computer Graphics Forum, 29(3):773-782, 2010.

[2] C. R. Butson, G. Tamm, S. Jain, T. Fogal, and J. Krüger. Evaluation of interactive visualization on mobile computing platforms for selection of deep brain stimulation parameters. IEEE Transactions on Visualization and Computer Graphics, 19(1):108-117, 2013.

[3] S. Castro, A. König, H. Löffelmann, and M. E. Gröller. Transfer function specification for the visualization of medical data. Technical report, Insitute of Computer Graphics, Vienna University of Technology, 1998.

[4] C. Correa and K.-L. Ma. Size-based transfer functions: A new volume exploration technique. IEEE Transactions on Visualization and Computer Graphics, 14(6):1380-1387, 2008.

[5] C. Correa and K.-L. Ma. Visibility-driven transfer functions. In IEEE Pacific Visualization Symposium (Pacific Vis 2009), pages 177-184, 2009.

[6] C. D. Correa and K.-L. Ma. Visibility histograms and visibility-driven transfer functions. IEEE Transactions on Visualization and Computer Graphics, 17(2):192-204, 2011.

[7] R. A. Drebin, L. Carpenter, and P. Hanrahan. Volume rendering. In Computer Graphics (Proceedings of SIGGRAPH 1988), pages 65-74, 1988.

[8] L. Gallo, G. De Pietro, A. Coronato, and I. Marra. Toward a natural interface to virtual medical imaging environments. In Working Conference on Advanced Visual Interfaces, pages 429-432. ACM, 2008.

[9] L. Gallo, G. De Pietro, and I. Marra. 3D interaction with volumetric medical data: experiencing the wiimote. In International Conference on Ambient Media and Systems, article 14, 2008.

[10] H. Guo, N. Mao, and X. Yuan. WYSIWYG (what you see is what you get) volume visualization. IEEE Transactions on Visualization and Computer Graphics, 17(12):2106-2114, 2011.

[11] H. Guo and X. Yuan. Local WYSIWYG volume visualization. In IEEE Pacific Visualization Symposium (PacificVis 2013), pages 65-72, 2013.

[12] T. He, L. Hong, A. Kaufman, and H. Pfister. Generation of transfer functions with stochastic search techniques. In IEEE Visualization, pages 227-234, 1996.

[13] C. Y. Ip, A. Varshney, and J. JaJa. Hierarchical exploration of volumes using multilevel segmentation of the intensity-gradient histograms. IEEE Transactions on Visualization and Computer Graphics, 18(12):23552363, 2012.

[14] G. Kindlmann and J. W. Durkin. Semi-automatic generation of transfer functions for direct volume rendering. In IEEE Symposium on Volume Visualization, pages 79-86, 1998.

[15] J. Kniss, G. Kindlmann, and C. Hansen. Multidimensional transfer functions for interactive volume rendering. IEEE Transactions on Visualization and Computer Graphics, 8(3):270-285, 2002.

[16] A. König and E. Gröller. Mastering transfer function specification by using volumepro technology. In Spring Conference on Computer Graphics, pages 279-286, 2001.

[17] S. Lindholm, P. Ljung, C. Lundström, A. Persson, and A. Ynnerman Spatial conditioning of transfer functions using local material distributions. IEEE Transactions on Visualization and Computer Graphics, 16(6):1301-1310, 2010.

[18] C. Lundström, P. Ljung, and A. Ynnerman. Extending and simplifying transfer function design in medical volume rendering using local histograms. Eurographics/IEEE VGTC Symposium on Visualization, pages
263-270, 2005

[19] C. Lundström, P. Ljung, and A. Ynnerman. The $\alpha$-histogram: Using spatial coherence to enhance histograms and transfer function design. In IEEE VGTC Symposium on Visualization (EuroVis 2006), pages 227-234, 2006.

[20] C. Lundström, P. Ljung, and A. Ynnerman. Local histograms for design of transfer functions in direct volume rendering. IEEE Transactions on Visualization and Computer Graphics, 12(6):1570-1579, 2006.

[21] C. Lundström, T. Rydell, C. Forsell, A. Persson, and A. Ynnerman. Multi-touch table system for medical visualization: Application to orthopedic surgery planning. IEEE Transactions on Visualization and Computer Graphics, 17(12):1775-1784, 2011.

[22] J. Marks, B. Andalman, P. A. Beardsley, W. Freeman, S. Gibson, J. Hodgins, T. Kang, B. Mirtich, H. Pfister, W. Ruml, K. Ryall, J. Seims, and S. Shieber. Design galleries: a general approach to setting parameters for computer graphics and animation. In SIGGRAPH '97, pages 389-400, 1997.

[23] N. Max. Optical models for direct volume rendering. IEEE Transactions on Visualization and Computer Graphics, 1(2):99-108, 1995.

[24] H. Pfister, B. Lorensen, C. Bajaj, G. Kindlmann, W. Schroeder, L. S Avila, K. Martin, R. Machiraju, and J. Lee. The transfer function bakeoff. IEEE Computer Graphics and Applications, 21(3):16-22, 2001.

[25] F. d. M. Pinto and C. M. D. S. Freitas. Two-level interaction transfer function design combining boundary emphasis, manual specification and evolutive generation. In Brazilian Symposium on Computer Graphics and Image Processing, pages 281-288, 2006.

[26] J.-S. Prassni, T. Ropinski, J. Mensmann, and K. Hinrichs. Shape-based transfer functions for volume visualization. In IEEE Pacific Visualization Symposium (PacificVis 2010), pages 9-16, 2010.

[27] J. L. Prauchner, J. L. Comba, et al. Two-level interaction approach for transfer function specification. In Brazilian Symposium on Computer Graphics and Image Processing, pages 265-272, 2005.

[28] B. Preim and C. P. Botha. Visual Computing for Medicine: Theory, Algorithms, and Applications. Morgan Kaufmann Publishers Inc., 2nd edition, 2013.

[29] C. Rezk-Salama, M. Keller, and P. Kohlmann. High-level user interfaces for transfer function design with semantics. IEEE Transactions on Visualization and Computer Graphics, 11(5):1021-1028, 2006.

[30] S. Roettger, M. Bauer, and M. Stamminger. Spatialized transfer functions. In Eurographics/IEEE VGTC Symposium on Visualization, pages 271278, 2005.

[31] T. Ropinski, J.-S. Prani, F. Steinicke, and K. Hinrichs. Stroke-based transfer function design. In IEEE/EG Symposium on Volume and Point-Based Graphics, pages 41-48, 2008.

[32] E. Shen, J. Xia, Z. Cheng, R. Martin, Y. Wang, and S. Li. Model-driven multicomponent volume exploration. The Visual Computer, 31(4):441454, 2015.

[33] Y. Wu and H. Qu. Interactive transfer function design based on editing direct volume rendered images. IEEE Transactions on Visualization and Computer Graphics, 13(5):1027-1040, 2007.

[34] Z. Zheng, N. Ahmed, and K. Mueller. iView: A feature clustering framework for suggesting informative views in volume visualization. IEEE Transactions on Visualization and Computer Graphics, 17(12):19591968,2011 